\title{
Complicated Persistent Peritonitis
}

\author{
Lindsay Roth, BA and Kumar Sarkar, MD
}

\section{INTRODUCTION}

Spontaneous bacterial peritonitis (SBP) is a common complication of end-stage liver disease. SBP can present with many symptoms such as abdominal pain, fever and altered mental status. ${ }^{1}$ The diagnosis of SBP is made when ascitic fluid from a paracentesis has an absolute neutrophil count (ANC) more than 250/uL, there is a positive ascitic fluid culture, and no secondary source of infection can be idenitifed. ${ }^{2}$ However, nearly $60 \%$ of patients with SBP have negative fluid cultures. ${ }^{3}$ These patients can still potentially have SBP and should be treated as such since in-hospital mortality ranges from 20-40\%. ${ }^{1,4}$ Conventional treatment for SBP includes a third-generation cephalosporin for five days, followed by lifetime prophylaxis most commonly with a fluoroquinolone. ${ }^{5}$ After 48 hours of antibiotic treatment, a repeat paracentesis should be performed. If the ANC does not decrease by at least $25 \%$, it is considered a therapeutic failure and the antibiotic should be changed. ${ }^{5}$ With early diagnosis and appropriate antibiotic regimen, SBP is treatable.

When therapy for SBP fails and the ascitic fluid ANC remains elevated, the peritonitis is considered persistent. Persistent peritonitis should raise suspicion for a secondary cause. An increase or lack of a significant decrease in the ANC in follow-up paracentesis should provoke a full work-up for secondary causes of peritonitis. ${ }^{1}$ Secondary causes include intra-abdominal abscess, bowel perforation, bile leak and malignancy. If a source is not found, persistent peritonitis should be treated with broad-spectrum antibiotics and/or antifungals early on. ${ }^{6}$ Furthermore, patients with a history of an ICU stay during the previous three months, antibiotic treatment during the previous three months, or a recent intervention in the hospital setting tend to be at higher risk for persistent, difficult to treat peritonitis? This includes resistance to third-generation cephalosporins and quinolones, which has been documented in 40-50\% of difficult to treat peritonitis cases. ${ }^{7}$ When patients have complications in their hospital course, difficult to treat peritonitis should be of concern.

\section{CASE PRESENTATION}

A 63-year-old transgender female with a two year history of decompensated NASH cirrhosis complicated by portal hypertension, esophageal variceal bleeding status post banding nine months prior, portal vein thrombosis, hepatic encephalopathy, COPD, sarcoidosis, anxiety and depression presented to a local hospital with septic shock secondary to cholangitis. The patient presented with fatigue, epigastric pain/distension and was hypotensive (80s/40s $\mathrm{mmHg}$ ) despite fluid resuscitation.

\begin{tabular}{|c|c|c|c|c|c|c|c|c|}
\hline $\begin{array}{c}\text { Date of } \\
\text { Paracentesis }\end{array}$ & $\begin{array}{c}\text { Date Range of } \\
\text { Antibiotic }\end{array}$ & Antiobiotic & $\begin{array}{l}\text { White Blood } \\
\text { Cell Count }\end{array}$ & \begin{tabular}{|c|} 
Absolute \\
Neutrophil Count
\end{tabular} & $\begin{array}{c}\text { Serum } \\
\text { Albumin }\end{array}$ & $\begin{array}{c}\text { Ascites } \\
\text { Albumin }\end{array}$ & $\begin{array}{c}\text { Serum-Ascites } \\
\text { Albumin Gradient }\end{array}$ & Culture \\
\hline $9 / 14$ & $9 / 10-9 / 17$ & $\begin{array}{l}\text { Piperacillin- } \\
\text { Tazobactam }\end{array}$ & 281 & 151 & 2.5 & & & Negative \\
\hline $9 / 20$ & $9 / 18-9 / 20$ & Levofloxacin & 1215 & 1081 & 3.8 & & & Negative \\
\hline $9 / 24$ & $9 / 21-10 / 2$ & Meropenem & 502 & 331 & 3.5 & 1.3 & 2.2 & Negative \\
\hline \multirow[t]{2}{*}{$10 / 1$} & & & 492 & 360 & 3.1 & 1.3 & 1.8 & Negative \\
\hline & $10 / 2$ & Anidulafungin & & & & & & \\
\hline $10 / 5$ & $10 / 6-10 / 10$ & $\begin{array}{l}\text { Meropenem \& } \\
\text { Anidulafungin }\end{array}$ & 492 & 373 & 3.2 & 1 & 2.2 & Negative \\
\hline $10 / 10$ & & & 127 & 58 & 2.8 & 0.9 & 1.9 & Negative \\
\hline $10 / 15$ & $10 / 11-10 / 25$ & Ciprofloxacin & 534 & 261 & 2.8 & & & Negative \\
\hline $10 / 22$ & & & 365 & 25 & 3.6 & 1.5 & 2.1 & Negative \\
\hline $10 / 26$ & $10 / 26-11 / 2$ & Cefuroxime & 339 & 23 & 4.1 & & & Negative \\
\hline $11 / 1$ & & & 298 & 35 & 4 & 2.1 & 1.9 & Negative \\
\hline $11 / 2$ & & & 292 & 26 & 3.9 & & & Negative \\
\hline
\end{tabular}

Figure 1. Paracentesis Results and Antibiotic Course 
Labs were notable for a leukocytosis ( $\left.23 \times 10^{\wedge} 9 / \mathrm{L}\right)$, thrombocytopenia $\left(36 \times 10^{\wedge} 9 / \mathrm{L}\right)$, hyperbilirubinemia (total bilirubin $8.1 \mathrm{mg} / \mathrm{dL}$ ), lactic acidosis (lactate 6.7 $\mathrm{mmol} / \mathrm{L}$ ), renal failure (creatinine $2.56 \mathrm{mg} / \mathrm{dL}$ ), and a MELD score of 37. The patient was started on vasopressors and broad-spectrum antibiotics. On imaging, the common bile duct was dilated to 19 millimeters with obstructing stones consistent for choledocholithiasis. She underwent endoscopic retrograde cholangiopancreatography (ERCP) with bile duct stenting and was started on Piperacillin-Tazobactam. After the procedure, she remained intubated for two days due to acute hypoxic respiratory failure. A day after extubation, she began to experience worsening abdominal distension and shortness of breath and therefore underwent large volume paracentesis, which was negative for SBP. Her liver function tests and total bilirubin continued to increase, so she underwent repeat ERCP and sphincterotomy with removal of a stone and the previously placed biliary stent.

Despite an eight-day course of Piperacillin-Tazobactam, followed by a three-day course of Levofloxacin for cholangitis, the patient underwent a therapeutic paracentesis and the ANC was found to be elevated to $1,081 / \mathrm{L}$ with negative cultures. She was started on Meropenem for SBP. She continued to have multiple therapeutic paracenteses, with persistently elevated ANCs despite antibiotic therapy (Figures 1 and 2). Cultures of the ascitic fluid remained negative through the entire course of peritonitis. Due to the persistently elevated ANCs, antifungal coverage with Anidulafungin was started as well. Through discussion with the infectious disease team, it was eventually decided to observe the patient off antibiotics and antifungal therapy in an effort to prevent intra-abdominal colonization of multidrug resistant organisms. However, while off antibiotics, she continued to have an elevated ANC without any culture growth and was therefore restarted on Meropenem and Anidulafungin. After five days of antibiotics, repeat paracentesis was finally negative for SBP. Her antibiotics were switched to Ciprofloxacin for SBP prophylaxis. After four days on the prophylactic antibiotic, a repeat paracentesis revealed an elevated ANC consistent with SBP. However, since the patient did not appear clinically infected, she was continued on prophylactic Ciprofloxacin. Unfortunately, a day later, the patient developed a pruritic rash on her trunk and legs consistent with a drug rash due to the Ciprofloxacin. Therefore, SBP prophylaxis was switched to Cefuroxime and repeat paracentesis was negative for SBP.

The patient did undergo a full work-up during her prolonged hospital course to rule out secondary causes of peritonitis. A CT scan of her abdomen and pelvis did not identify an intra-abdominal abscess or bowel perforation. Abdominal ultrasound was negative for a bile leak and testicular ultrasound was negative for testicular torsion or epididymitis. A HIDA scan was negative for cholecystitis and ascitic fluid cytology was negative for malignancy. Fortunately, the patient was able to be re-listed on the liver transplant list following clearance of the infection and she successfully underwent liver transplant shortly thereafter.

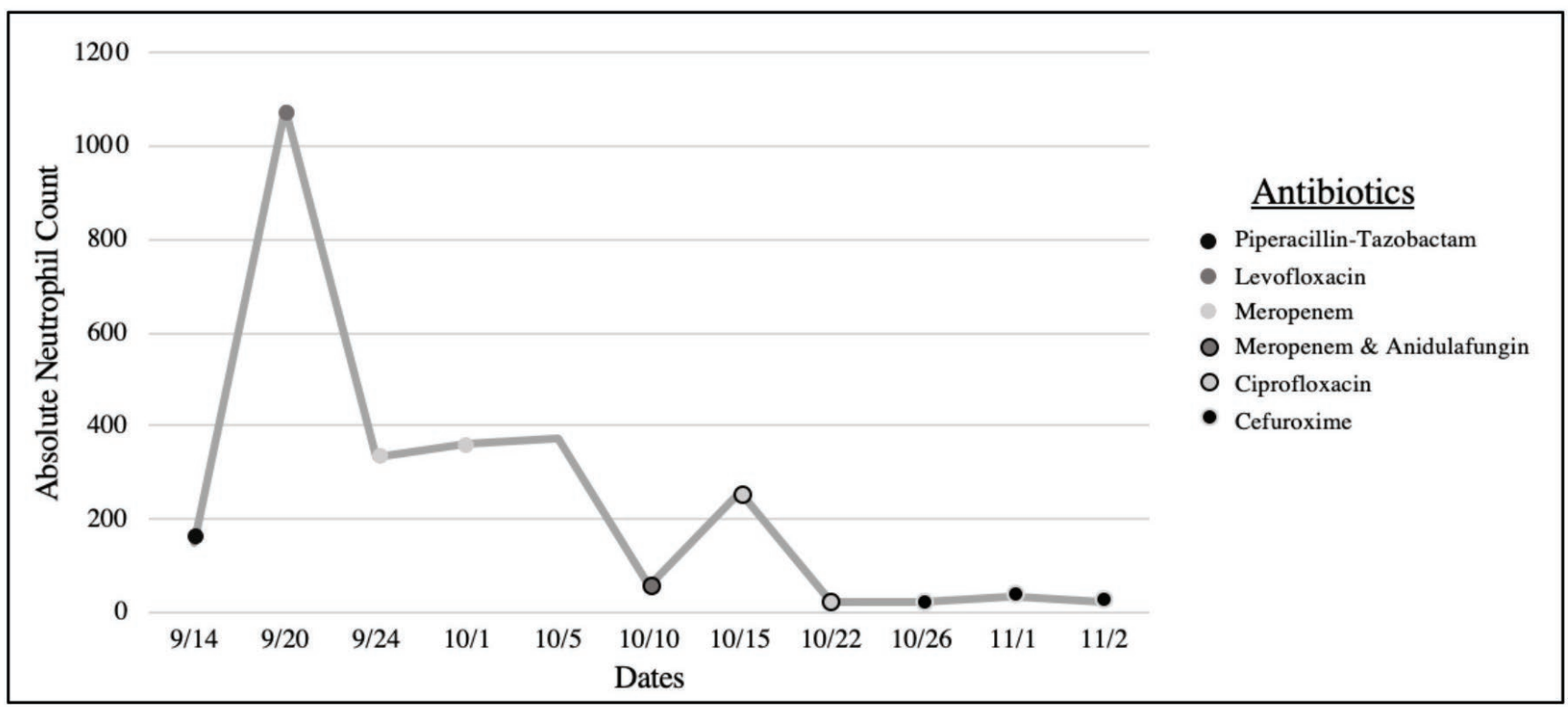

Figure 2. Timeline of Antibiotics and Paracentesis Results 


\section{DISCUSSION}

The diagnosis of SBP is made when paracentesis fluid analysis has an ANC of more than 250/ uL, a positive fluid culture and no secondary source of an intra-abdominal infection. ${ }^{2}$ While our patient fulfilled two of these criteria, she also had multiple risk factors for difficult to treat peritonitis. Some of the risk factors for difficult to treat peritonitis include an ICU admission during the previous three months, antibiotic treatment during the previous three months, and a recent intervention in the hospital setting, all of which our patient had.?

Management of persistent peritonitis should include an extensive work-up for secondary peritonitis. ${ }^{1}$ Once a secondary cause is ruled out, persistent peritonitis should be approached by broadening antibiotics and starting an antifungal. ${ }^{6}$ However, this can lead to development of multidrug resistant organisms. Further research is needed on treatment for persistent peritonitis to develop definitive management, while considering multidrug resistant organisms.

\section{REFERENCES}

1. Rimola A, Garcia-Tsao G, Navasa M, Piddock L, Planas R, Bernard B. Diagnosis, treatment and prophylaxis of spontaneous bacterial peritonitis: a consensus document. J Hepatol. 2000; 32:142-153.

2. Such J, Runyon BA. Spontaneous Bacterial Peritonitis. Clin Infect Dis. 1998; 27:669-674

3. Nobre SR, Cabral JEP, Gomes JJF, Leitão MC. In-hospital mortality in spontaneous bacterial peritonitis: A new predictive model. Eur J Gastroenterol Hepatol. 2008; 20(12):1176-1181. doi:10.1097/MEG.0b013e32830607a2.

4. Thuluvath PJ, Morss S, Thompson R. Spontaneous bacterial peritonitisin-hospital mortality, predictors of survival, and health care costs from 1988 to 1998. Am J Gastroenterol. 2001; 96:1232-1236

5. Alaniz C, RE R. Spontaneous bacterial peritonitis: a review of treatment options. P\&T A Peer-Reviewed J Manag Care Formul Manag. 2009; 34(4):204-213

6. Desai AP, Reau N, Reddy KG, et al. Persistent spontaneous bacterial peritonitis: A common complication in patients with spontaneous bacterial peritonitis and a high score in the model for end-stage liver disease. Therap Adv Gastroenterol. 2012; 5(5):275-283.

7. Shalimar SKA. Difficult to treat spontaneous bacterial peritonitis. Trop Gastroenterol. 2013; 34(1):7-13. 